\title{
Editorial
}

\section{Fair Optimization and Networks: Models, Algorithms, and Applications}

\author{
Wlodzimierz Ogryczak, ${ }^{1}$ Hanan Luss, ${ }^{2}$ Dritan Nace, ${ }^{3}$ and Michał Pióro ${ }^{4,5}$ \\ ${ }^{1}$ Institute of Control and Computation Engineering, Warsaw University of Technology, 00-665 Warsaw, Poland \\ ${ }^{2}$ Department of Industrial Engineering and Operations Research, Columbia University, New York, NY 10027, USA \\ ${ }^{3}$ Laboratoire Heudiasyc, Université de Technologie de Compiègne, 60203 Compiègne, France \\ ${ }^{4}$ Department of Electrical and Information Technology, Lund University, 22100 Lund, Sweden \\ ${ }^{5}$ Institute of Telecommunications, Warsaw University of Technology, 00-665 Warsaw, Poland
}

Correspondence should be addressed to Wlodzimierz Ogryczak; w.ogryczak@elka.pw.edu.pl

Received 21 August 2014; Accepted 21 August 2014; Published 10 September 2014

Copyright (C) 2014 Wlodzimierz Ogryczak et al. This is an open access article distributed under the Creative Commons Attribution License, which permits unrestricted use, distribution, and reproduction in any medium, provided the original work is properly cited.

Optimization models related to the design and evaluation of system policies are mainly focused on efficiency metrics such as the response time, queue length, throughput, and cost. However, in systems which serve many users there is a need to respect some fairness rules while looking for the overall efficiency [1]. Essentially, fairness is an abstract sociopolitical concept that implies impartiality, justice, and equity. In order to ensure fairness in a system, all system entities have to be adequately provided with the system's services. Nevertheless, fair treatment of all entities does not imply equal allocation of services due to constraints imposed on the system by various entities and by the environment. Within the system analysis, fairness was usually quantified with the so-called inequality measures such as variance and mean absolute difference [2]. Unfortunately, direct minimization of typical inequality measures contradicts the maximization of individual outcomes and it also may lead to inefficient designs [3]. Yet, fair optimization with a preference structure that complies with both efficiency and the equitability can be used to generate a variety of fair and efficient solutions $[4,5]$. The so-called lexicographic maximin (or minimax) optimization concept [6-9] (and a closely related max-min fairness optimization concept [10]) extends max-min optimization models and is widely applied to various systems. A lexicographic maximin objective optimizes the worst performance among all system entities, followed by optimizing the second worst performance without degrading the worst one, and so forth. However, this may cause a dramatic worsening of the overall system efficiency. Therefore, several other fair optimization models, which compromise between fairness and overall system efficiency, have been extensively analyzed.

The issue of fairness is widely recognized in location and allocation analysis of public services, where the clients of a system are entitled to fair treatment according to community regulations. The need of fair optimization arises also in more general problems of resource allocation [11], for example, in problems related to various networking systems. Fair network optimization issues are in focus of diverse applications and optimization problems in communication networks [12, 13]. These issues are closely related to situations where it is desirable to achieve an equitable allocation for given resources shared by competing traffic demands. Fairness, and more specifically, lexicographic maximin optimization $[6,8,14]$, max-min fairness [10], and proportional fairness [15] are widely studied in the communication network literature, especially in relation to bandwidth allocation and rate adaptation and congestion control in TCP (transmission control protocol) networks. At the same time, these concepts are widely applicable in different settings in network optimization and more specifically in the multicommodity flow network related applications. 
The presented special issue strives to serve as a platform to present advances in the field. We have invited potential authors to submit original research articles that propose new models, algorithms, and applications of fair network optimization. Out of a total number of 36 papers that were submitted, after a strict review process, 11 high-quality papers are published. The special issue contains one review paper and ten research papers that consider several closely related and interesting topics. In what follows we briefly describe the published contributions.

The review paper "Fair optimization and networks: a survey" authored by the editors jointly with A. Tomaszewski overviews fair optimization models and algorithms supporting efficient and fair resource allocation applicable to problems involving network flows that express realizations of competing activities. The presentation applies to communication systems, power distribution systems, transportation systems, logistics systems, and so forth. A particular focus is on allocation problems related to communication networks since in this area the fair optimization concepts have been extensively developed and widely applied.

The paper "Optimization of power allocation for a multibeam satellite communication system with interbeam interference" by $\mathrm{H}$. Wang et al. considers power allocation among multiple beams in satellite communication that compromises between maximizing the total system capacity and providing a fair power allocation among the beams. The model is formulated as a nonlinear optimization problem that considers interbeam interferences. Locally optimal solutions are obtained by employing an iterative procedure that is based on duality theory. Simulation results demonstrate the effectiveness of the proposed allocation algorithm.

The paper "Constructing fair destination-oriented directed acyclic graphs for multipath routing" by K. KalinowskaGórska and F. S. Donado examines the issue of determining candidate paths between node pairs that can potentially be used in multicommodity network flow algorithms with applications in communication networks. The paper determines a subset of candidate directed paths from many sources to a single root node such that the number of paths assigned to each source-root pair is considered fair. This is achieved by formulating and solving an optimization problem with integer variables and a lexicographic maximin objective. The authors provide numerical results that compare their method to several existing methods.

In the paper "Max-min fair link quality in WSN based on SINR" A. Gogu et al. address the problem of scheduling max-min fair link transmissions in wireless sensor networks, jointly with transmission power assignment. Given a set of concurrently transmitting links, the considered optimization problem seeks for transmission power levels at the nodes so that the signal-to-interference and noise ratio (SINR) values of active links satisfy the max-min fairness property. By guaranteeing a fair transmission medium (in terms of SINR), other network requirements, such as the scheduling length, the throughput (directly dependent on the number of concurrent links in a time slot), and energy savings (no collisions and retransmissions), can be directly controlled.
In his paper "Design of optical wireless networks with fair traffic flows," A. Tomaszewski studies a network design problem under variable/uncertain capacity. The work is motivated by practical considerations in wireless networks such as difficulties in traffic transmissions caused by bad weather conditions. In addition to the nominal network state where capacities have their nominal values, the author introduces a set of failure states where capacities are fixed to lower values. Then, the demands are routed in the network with reduced link capacities by decreasing the packet rate fairly, provided that it does not go below some given threshold.

The paper "Max-min fairness in WMNs with interference cancelation using overheard transmissions" written by M. Żotkiewicz deals with a recent topic related to wireless mesh networks, namely, to the interference cancellation (IC) technique. The idea is to use IC even for cases with high SINR values taking advantage of overheard traffic; the proposed idea is illustrated with an example. A relevant MIP model for determining scheduling and maximal throughput in a network under these hypotheses is proposed. Simulation results illustrate the benefits of the proposed approach.

The paper "Threshold accepting heuristic for fair flow optimization in wireless mesh networks," by J. Hurkała and T. Śliwiński, focuses once again on wireless mesh networks and reports an application of a list-based threshold accepting (LBTA) heuristic that maximizes total throughput while preserving fairness among network flows. The authors use weighted ordered weighted averaging (WOWA) operator to model fairness, while LBTA heuristic is used instead of simulated annealing (SA). Numerical results show that LBTA performs much better than SA in terms of the computing time.

In "Fair optimization of video streaming quality of experience in LTE networks using distributed antenna systems and radio resource management," E. Yaacoub and Z. Dawy focus on QoE (quality of experience) used to measure the multimedia experience of mobile users. The authors propose QoE metrics in order to capture the overall performance of the radio resource management (RRM) algorithms in terms of video quality perceived by the end users. Instead of investigating QoE on the link level, they study metrics for assessing the QoE performance over the whole network, taking into account fairness constraints. They consider the use of distributed antenna systems (DAS) to enhance the performance and show by numerical tests that combining DAS and fair RRM algorithms can lead to significant and fair QoE enhancements for all the users in the network.

The paper "An approximation algorithm for the facility location problem with lexicographic minimax objective" by L. Buzna et al. presents a new approximation algorithm to the lexicographic minimax optimum of the discrete facility location problem. The approach uses algorithms originally designed to solve the $\mathrm{p}$-median problem and it allows for finding equitable location of facilities serving a large number of customers.

In the paper " $A$ fairness relation based on the asymmetric Choquet integral and its application in network resource allocation problems," A. Honda and M. Köppen study a recent 
problem of network resource allocation where pairs of users could be in a favourable situation, given that the allocation scheme is refined by some add-on technology. They propose a computational approach based on the framework of relational optimization. For representing different weightings for different pairs of users, a binary relation using the asymmetric Choquet integral is introduced as the most suitable approach to represent fairness.

The paper "Price of fairness on networked auctions" by M. Kaleta examines execution of auctions under certain networking constraints applicable, for example, in the electricity, communications, and water allocation problems. In classical auctions, a marginal pricing principle is usually applied, which provides a solution acknowledged by all participants as fair. In the case of networked auctions the fairness conditions can be disrupted and the uniform market pricing cannot be used. The paper focuses on multicommodity networks with sealed-bid auctions and shows that the minimal price of fairness can be achieved if the unconstrained market price settlements are adjusted with additional node-dependent prices paid at each node.

\section{Acknowledgments}

The editors would like to thank all the authors and reviewers for their valuable contribution to this special issue. The Polish editors would also like to kindly acknowledge the role of the National Science Centre (Poland) who sponsored their activities related to preparing this special issue through the Grant 2011/01/B/ST7/02967 "Integer programming models for joint optimization of link capacity assignment, transmission scheduling, and routing in fair multicommodity flow networks."

Wlodzimierz Ogryczak
Hanan Luss
Dritan Nace
Michat Pióro

\section{References}

[1] H. Luss, Equitable Resource Allocation: Models, Algorithms, and Applications, John Wiley \& Sons, Hoboken, NJ, USA, 2012.

[2] A. B. Atkinson, "On the measurement of inequality," Journal of Economic Theory, vol. 2, pp. 244-263, 1970.

[3] W. Ogryczak, "Inequality measures and equitable locations," Annals of Operations Research, vol. 167, pp. 61-86, 2009.

[4] M. M. Kostreva and W. Ogryczak, "Linear optimization with multiple equitable criteria," RAIRO Operations Research, vol. 33, no. 3, pp. 275-297, 1999.

[5] M. M. Kostreva, W. Ogryczak, and A. Wierzbicki, "Equitable aggregations and multiple criteria analysis," European Journal of Operational Research, vol. 158, no. 2, pp. 362-377, 2004.

[6] H. Luss, "On equitable resource allocation problems: a lexicographic minimax approach," Operations Research, vol. 47, no. 3, pp. 361-378, 1999.

[7] H. Luss and D. R. Smith, "Resource allocation among competing activities: a lexicographic minimax approach," Operations Research Letters, vol. 5, no. 5, pp. 227-231, 1986.
[8] D. Nace and J. B. Orlin, "Lexicographically minimum and maximum load linear programming problems," Operations Research, vol. 55, no. 1, pp. 182-187, 2007.

[9] W. Ogryczak, "On the lexicographic minimax approach to location problems," European Journal of Operational Research, vol. 100, no. 3, pp. 566-585, 1997.

[10] D. Nace and M. Pióro, "Max-min fairness and its applications to routing and load-balancing in communication networks: a tutorial," IEEE Communications Surveys and Tutorials, vol. 10, no. 4, pp. 5-17, 2008.

[11] W. Ogryczak, "Multicriteria models for fair resource allocation," Control and Cybernetics, vol. 36, no. 2, pp. 303-332, 2007.

[12] D. Bertsekas and R. Gallager, Data Networks, Prentice-Hall, Englewood Cliffs, NJ, USA, 1987.

[13] M. Pióro and D. Medhi, Routing, Flow and Capacity Design in Communication and Computer Networks, Morgan Kaufmann, San Francisco, Calif, USA, 2004.

[14] W. Ogryczak, M. Pióro, and A. Tomaszewski, "Telecommunications network design and max-min optimization problem," Journal of Telecommunications and Information Technology, vol. 2005, no. 3, pp. 43-56, 2005.

[15] F. P. Kelly, A. K. Maulloo, and D. K. H. Tan, "Rate control for communication networks: shadow prices, proportional fairness and stability," Journal of the Operational Research Society, vol. 49, no. 3, pp. 237-252, 1998. 


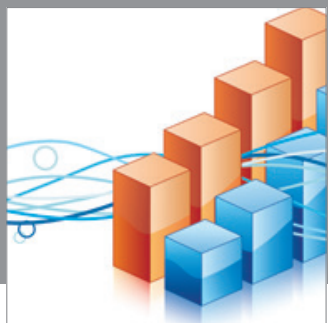

Advances in

Operations Research

mansans

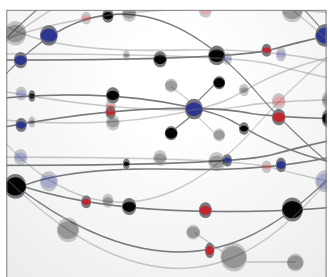

The Scientific World Journal
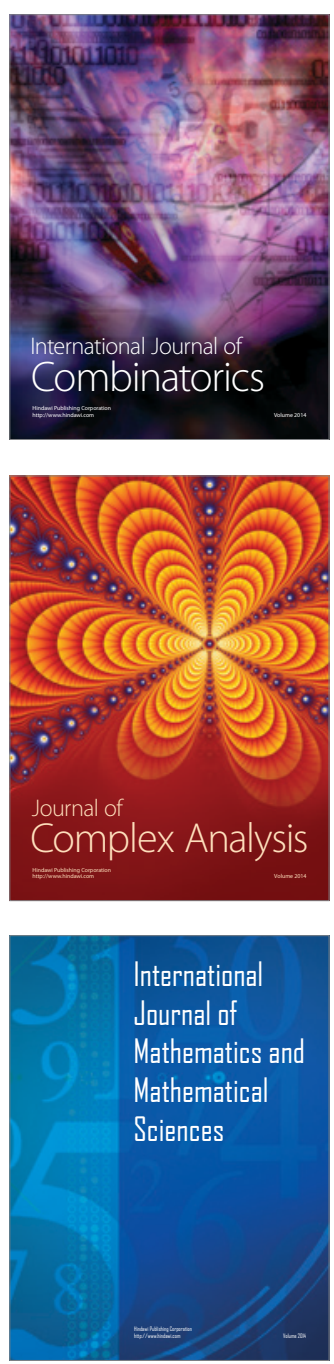
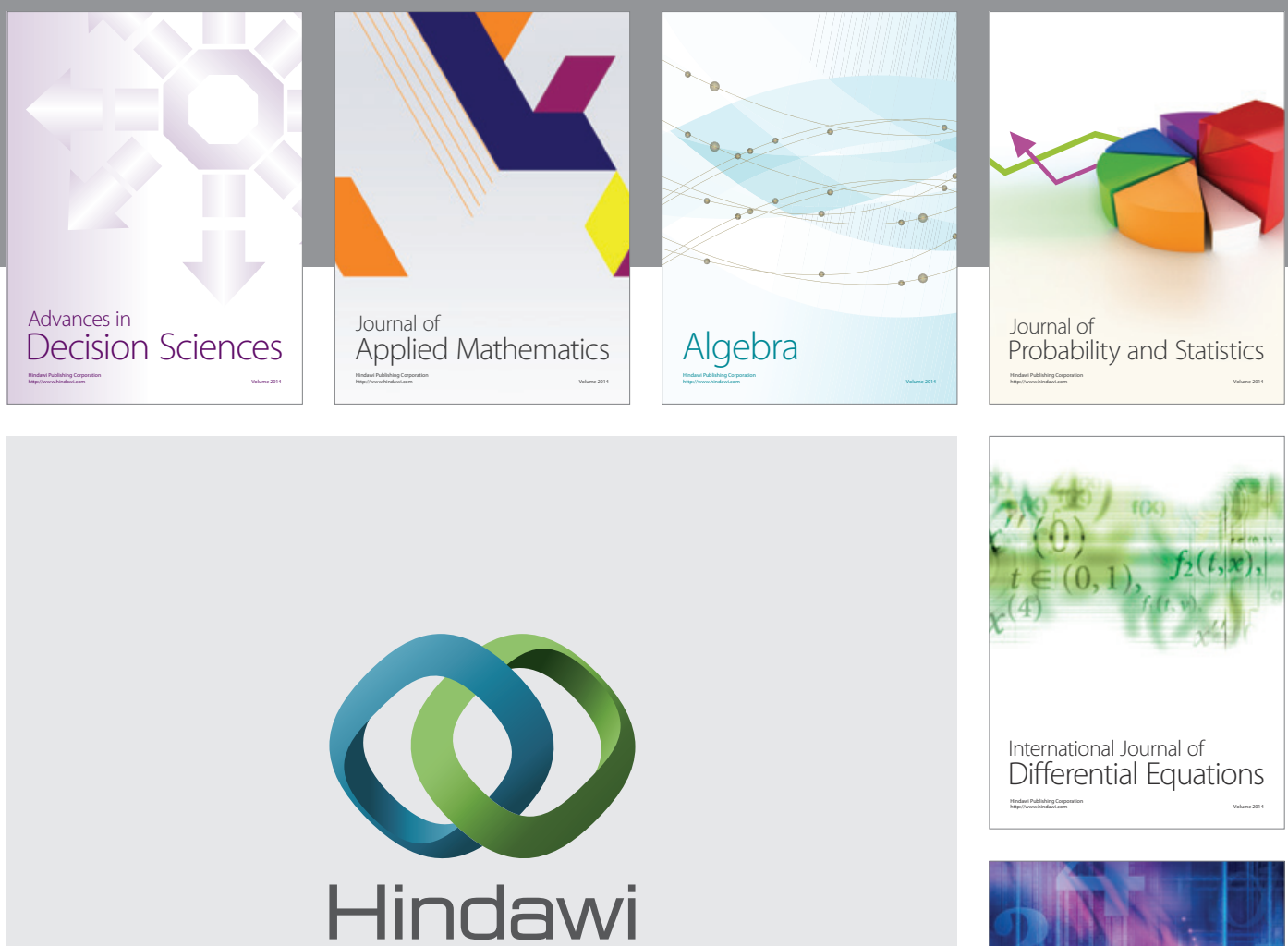

Submit your manuscripts at http://www.hindawi.com
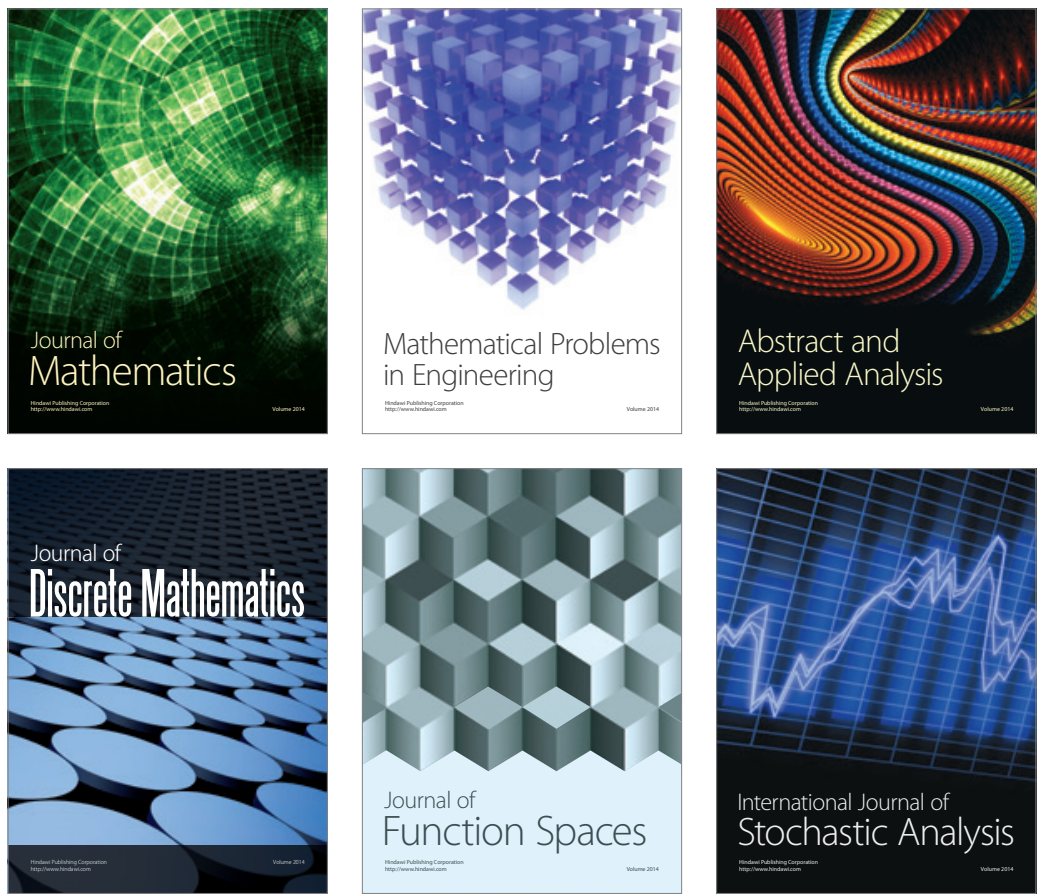

Journal of

Function Spaces

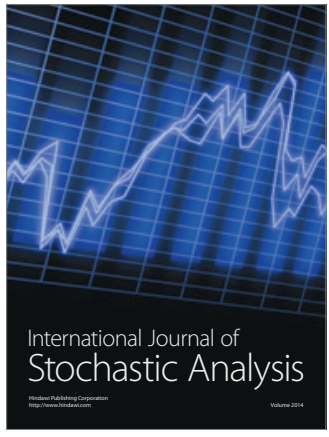

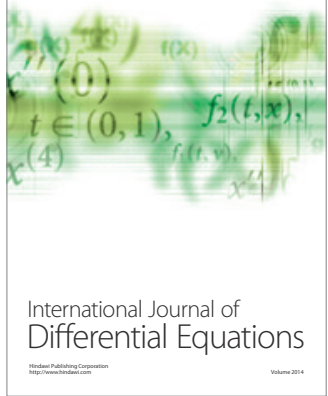
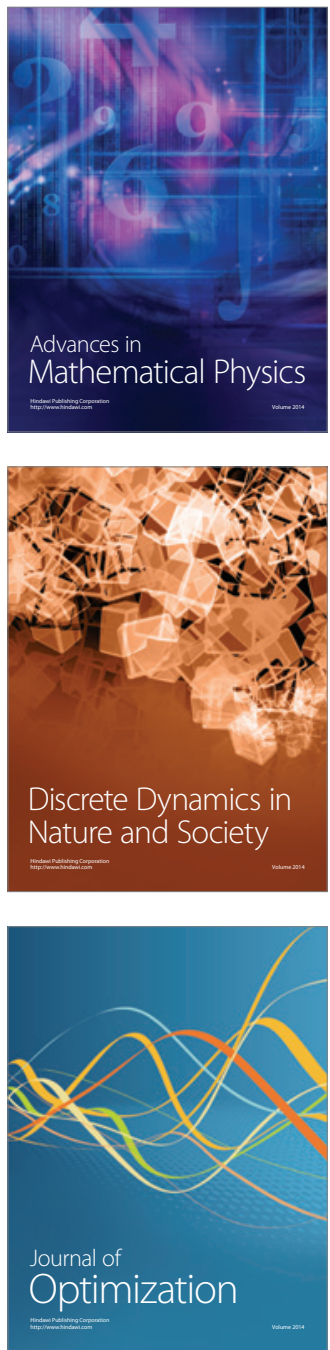